\title{
Angiopoietin-like Protein 2 Is an Important Facilitator of Inflammatory Carcinogenesis and Metastasis
}

\author{
Jun Aoi ${ }^{1,2}$, Motoyoshi Endo ${ }^{1}$, Tsuyoshi Kadomatsu ${ }^{1}$, Keishi Miyata ${ }^{1}$, Masahiro Nakano ${ }^{1}$, Haruki Horiguchi ${ }^{1}$, \\ Aki Ogata ${ }^{1,2}$, Haruki Odagiri ${ }^{1}$, Masato Yano ${ }^{1}$, Kimi Araki ${ }^{4}$, Masatoshi Jinnin ${ }^{2}$, Takaaki Ito ${ }^{3}$, \\ Satoshi Hirakawa ${ }^{5}$, Hironobu $\mathrm{Ihn}^{2}$, and Yuichi Oike ${ }^{1}$
}

\begin{abstract}
Chronic inflammation plays important roles at different stages of cancer development, including carcinogenesis, tumor invasion, and metastasis, but molecular mechanisms linking inflammation to cancer development have not been fully clarified. Here, we report that expression of angiopoietin-like protein 2 (Angptl2), recently identified as a chronic inflammation mediator, is highly correlated with the frequency of carcinogenesis in a chemically induced skin squamous cell carcinoma (SCC) mouse model. Furthermore, Angptl2 expression in SCC is highly correlated with the frequency of tumor cell metastasis to distant secondary organs and lymph nodes. When SCC was induced in transgenic mice expressing Angptl2 in skin epithelial cells, epithelial-to-mesenchymal transitions in SCC as well as tumor angiogenesis and lymphangiogenesis were significantly increased, resulting in increased tumor cell metastasis and shortened survival compared with wild-type mice. Conversely, in a chemically induced SCC mouse model, carcinogenesis and metastasis were markedly attenuated in Angptl2 knockout mice, resulting in extended survival compared with wild-type mice. Overall, we propose that Angptl2 contributes to increased carcinogenesis and metastasis and represents a novel target to antagonize these pathologies. Cancer Res; 71(24); 7502-12. (C2011 AACR.
\end{abstract}

\section{Introduction}

Cancer is an increasingly prevalent medical and social problem and remains a major cause of mortality (1). Therefore, the identification of mechanisms underlying its development is essential to develop new therapeutic approaches. Recently, the concept that chronic inflammation plays an important role at different stages of cancer development, including carcinogenesis, invasion, and metastasis, has emerged (2); it is well established that inflammation induced by bacterial and viral infections increases cancer risk, as does chronic inflammation induced by environmental exposure, including tobacco smoking and inhaled pollutants, such as silica and asbestos (3-5). It has also been recently reported that chronic and often subclinical levels of inflammation-for example, obesity-induced inflammation-may increase cancer risk (6). In fact, obesity,

Authors' Affiliations: Departments of ${ }^{1}$ Molecular Genetics, ${ }^{2}$ Dermatology and Plastic Surgery, ${ }^{3}$ Pathology and Experimental Medicine, Graduate School of Medical Sciences, ${ }^{4}$ CARD, Kumamoto University, Kumamoto; and ${ }^{5}$ Department of Dermatology, Hamamatsu University, Hamamatsu, Shizuoka, Japan

Note: Supplementary data for this article are available at Cancer Research Online (http://cancerres.aacrjournals.org/).

J. Aoi and M. Endo contributed equally to this study.

Corresponding Author: Yuichi Oike, Department of Molecular Genetics, Kumamoto University, 1-1-1 Honjo, Kumamoto 860-8556, Japan. Phone: 8196-373-5140; Fax: 81-96-373-5145; E-mail: oike@gpo.kumamoto-u.ac.jp

doi: 10.1158/0008-5472.CAN-11-1758

(C2011 American Association for Cancer Research. which increases cancer risk by 1.6-fold (7), can cause chronic inflammation, which can then promote carcinogenesis of the liver and pancreas (8). Recently, we found that angiopoietinlike protein 2 (Angptl2) is a causative mediator of chronic inflammation in obesity, and its related metabolic abnormalities (9). In obesity, Angptl2 is secreted by adipose tissue, and its expression is increased by obesity-related pathologic conditions, including hypoxia and endoplasmic reticulum (ER) stress (9). Both hypoxic stress and ER stress are commonly induced in cancer tissues, particularly in cancer progression and metastasis (10), suggesting a role of Angptl2 in these processes. Along these lines, it has been reported that increased levels of Angptl2 derived from tumor- or cancerassociated fibroblasts (CAF), respectively, in cancer tissues refractory to anti-VEGF therapy, which when combined with chemotherapy, is efficacious in treating several human cancers (11). Integrins, which act as functional receptors for Angptl2 in endothelial cells and monocytes/macrophages $(9,12)$, are also expressed on several cancer cells, in which they regulate tumor cell growth, survival, and invasion $(13,14)$. Angptl2 is expressed in some tumor cell types (15). Thus, cancer cell- and/or CAF-derived Angptl2 may be a critical factor in cancer development.

Carcinogenesis consists of 2 different processes: "preneoplastic change" and "malignant conversion" (16). The protocol used to chemically induce carcinogenesis in mouse skin consists of 2-stage application of chemicals to the skin, resulting in cutaneous tumors $(17,18)$. A single application of the initiator mutagen 7,12-dimethylbenzanthracene (DMBA) is followed by repeated applications of phorbol 12-myristate 13-acetate 
(PMA) (17). In this model $(19,20)$, papilloma formation as epidermal dysplasia, which represents a "preneoplastic change" in skin epidermal cells, is caused by DMBA-induced mutations in the H-ras gene, and the degree of "preneoplastic change" can be estimated by the number and size of papillomas, whose growth is stimulated by subsequent, repeated PMA applications. Finally, "malignant conversion" is accelerated by mutations in the $p 53$ gene brought on by subsequent serial PMA treatment (16) and is reflected in the rate of conversion from large papillomas to squamous cell carcinoma (SCC).

In this study, we found that Angptl2 expression was induced during chemically induced skin SCC development, whereas Angptl2 expression was faintly detected in normal skin tissues, suggesting a possible role for Angptl2 in chemically induced skin SCC. Therefore, we examined skin carcinogenesis and cancer progression by comparing chemically induced skin SCC in 3 mouse genotypes: K14-Angptl2 transgenic mice (K14Angptl2 Tg), Angptl2 knockout mice (Angptl2 KO), and respective wild-type littermates. Our finding in this study shows the first evidence that Angptl2 contributes to both increased susceptibility to "preneoplastic change" and "malignant conversion" in carcinogenesis and enhanced cancer cell metastasis, suggesting that Angptl2 represents a new target to block cancer development.

\section{Materials and Methods}

\section{Mice}

Only male mice were used for the experiments. For chemical carcinogenesis assays, K14-Angptl2 Tg (9) and Angptl2 KO (9) were backcrossed to the $\mathrm{FVB} / \mathrm{N}$ strain for more than 10 generations. All experiments were conducted according to guidelines of the Institutional Animal Committee of Kumamoto University.

\section{Immunoblot analysis}

Cells were homogenized in lysis buffer $(10 \mathrm{mmol} / \mathrm{L} \mathrm{NaF}, 1$ $\mathrm{mmol} / \mathrm{L} \mathrm{Na} \mathrm{VO}_{4}, 1 \mathrm{mmol} / \mathrm{L}$ EDTA, $300 \mathrm{mmol} / \mathrm{L} \mathrm{NaCl}$, $50 \mathrm{mmol} / \mathrm{L}$ Tris- $\mathrm{HCl}, 1 \%$ Triton X-100, pH 7.5). Extracts derived from supernatants were subjected to SDS-PAGE, and proteins were electrotransferred to nitrocellulose membranes. Immunodetection was carried out using an ECL Kit (Amersham) according to the manufacturer's protocol. Polyclonal antibodies against Smad2/3 and phospho-Smad2 (138D4) and monoclonal antibodies (mAb) against Hsc70 (B-6) were obtained from Cell Signaling and Santa Cruz, respectively.

\section{Immunohistochemical staining}

Tumors were fixed by perfusion with $4 \%$ paraformaldehyde in PBS (pH 7.4), washed in PBS for 15 minutes, dehydrated through a graded series of ethanol and xylene, and embedded in a single paraffin block. Sections $(5 \mu \mathrm{m})$ were cut, air-dried, deparaffinized, and pretreated with $5 \mathrm{mmol} / \mathrm{L}$ periodic acid for 10 minutes at room temperature to inhibit endogenous peroxidase. Specimens were incubated for 1 hour with 50- to 100fold diluted polyclonal antibody against Angptl2 (9), Keratin 5 (Covance), Keratin 14 (Covance), Snail (Cell Signaling), or Slug (Cell Signaling), or mAb against E-cadherin (BD Biosciences) or
$\mathrm{N}$-cadherin (BD Biosciences) and then washed 3 times with PBS for 5 minutes. Sections were incubated with biotinylated anti-mouse IgG or anti-rabbit IgG (1:200 dilution; Vector Laboratories). Immunostaining was done using the peroxidase-labeled avidin-biotin complex method (1:100 dilution; Dako). Sections were counterstained with hematoxylin.

\section{Calculation of survival data}

The Kaplan-Meier log-rank test was applied to analyze mouse survival data using JMP7 software (SAS Institute). A $P$ value of less than 0.05 was considered significant.

\section{Quantification of extent of metastasis}

Tumor cells within lung, liver, spleen tissues, and lymph nodes were visualized in tissue slices stained with hematoxylin and eosin (H\&E) and histologically identified by 2 independent investigators. The number and area of the metastatic region in lung tissue were quantified by evaluating 10 slices from K14Angptl2 Tg, Angptl2 KO, and respective wild-type littermates. The area was quantified as pixels using the BZ-H1M system (Keyence).

\section{Results}

\section{Expression of Angpt12 is induced in chemically promoted squamous cell carcinoma}

Increased Angptl2 mRNA expression was detected in skin tissues of chemically induced papilloma and SCC compared with that seen in normal skin tissues before treatment (Supplementary Fig. S1). Abundant Angptl2 protein was also observed in induced mouse skin SCC, whereas Angptl2 was weakly expressed in normal skin tissues before treatment (Supplementary Fig. S2A). We next asked which cell types express Angptl2. As shown in Supplementary Fig. S2B and Fig. S3, Angptl2 expressed faintly in keratinocytes of the interfollicular epidermis, basal keratinocytes, suprabasal keratinocytes, and hair follicles. In contrast, Angptl2 was abundantly expressed not only in tumor cells at the primary site but at metastatic regions, including distant secondary lung organ and lymph nodes (Supplementary Fig. S2C-E). Normal lymph node and lung tissues showed little Angptl2 expression (Supplementary Fig. S4).

\section{Accelerated carcinogenesis in skin tissues constitutively expressing Angptl2}

As shown in Fig. 1A, the ears of K14-Angptl2 Tg were redder in color than ears of wild-type mice, indicative of constitutive inflammation. We found that expression levels of the inflammatory markers $I L-6$ and $I L-1 \beta$ were significantly increased in skin tissues of K14-Angptl2 Tg compared with wild-type mice (Supplementary Fig. S5A). K14-Angptl2 Tg did not show papillomas or SCC during their lifespan, indicating that Angptl2 overexpression is not sufficient to cause carcinogenesis. To investigate whether Angptl2 overexpression affects chemically induced carcinogenesis, K14-Angptl2 Tg were subjected to the skin carcinogenesis regimen. This model requires application of both DMBA and PMA (16-18). PMA treatment alone induced Angptl2 expression in wild-type mice (Supplementary 


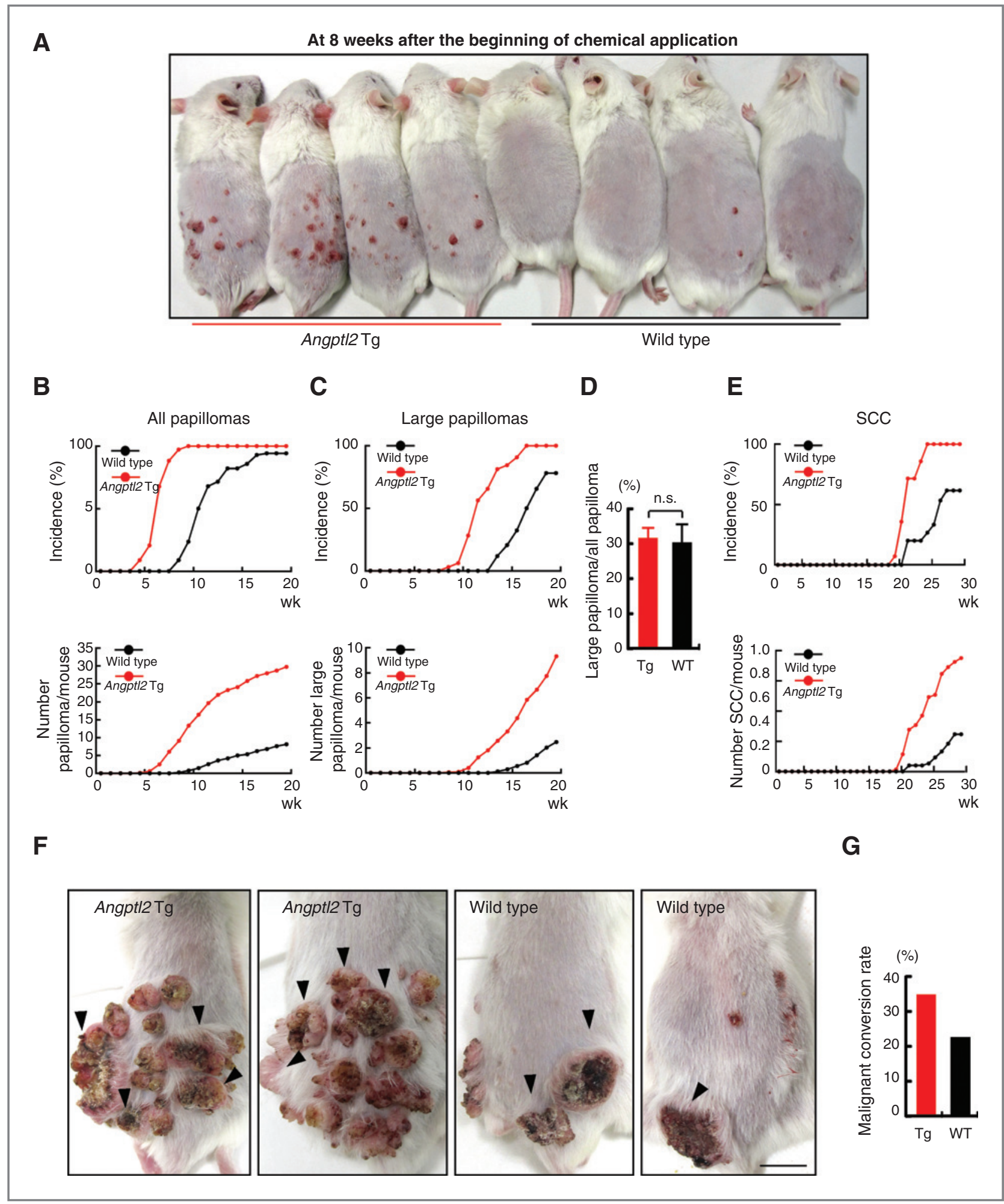

Figure 1. K14-Angpt/2 transgenic mice exhibit accelerated and increased skin carcinogenesis. A, photograph of skin of K14-Angpt/2 Tg (left) and wild-type mice (right) 8 weeks after the first chemical application. B and C, top graphs, the percentage of mice with papillomas as the incidence. Bottom graphs, number of all detectable (>1 mm; B) and large ( $>3 \mathrm{~mm}$; C) papillomas from K14-Angpt/2 Tg $(n=34$; red circles) and wild-type mice $(n=34$; black circles) $P<0.001$ from weeks 14 to 20 (bottom graph in B), $P<0.001$ from weeks 9 to 20 (bottom graph in C). $D$, proportion of large relative to all detectable papillomas in K14-Angpt/2 Tg and wild-type mice. n.s., not significant. Data represent means \pm SEM. E, increased incidence of SCC (top graph) and number of SCC per mouse (bottom graph, $P<0.001$ after week 21$)$ in K14-Angpt/2 Tg ( $n=33$; red circles) compared with wild-type mice ( $n=34$; black circles). F, photograph of SCC on K14-Angpt/2 Tg (left) and wild-type mice (right) 8 weeks after first diagnosis of SCC. Arrowheads indicate SCC. Scale bars, $10 \mathrm{~mm}$. G, comparison of ratio of malignant conversion of large papillomas to SCC in K14-Angpt/2 Tg and wild-type mice. WT, wild type. 
Fig. S6), but we observed no papillomas or SCC at any time in K14-Angptl2 Tg or wild-type mice treated with DMBA only or PMA only (data not shown), indicating that Angptl2 overexpression cannot cause a "preneoplastic change." At 8 weeks after beginning chemical treatment of both DMBA and PMA, numerous papillomas were detected in K14-Angptl2 Tg, whereas only a few were seen in wild-type mice (Fig. 1A). K14-Angptl2 $\mathrm{Tg}$ also showed accelerated formation of skin papillomas, with an average latency of 7 weeks after the beginning of chemical application, as compared with 10 weeks for wild-type mice (top graph in Fig. 1B). The number of papillomas was also significantly greater in K14-Angptl2 Tg (bottom graph in Fig. 1B). At 20 weeks after beginning of chemical treatment, K14-Angptl2 Tg exhibited an average of 29.8 papillomas per mouse, compared with 8.1 in wild-type mice $(P<0.001)$. When only large papillomas (diameter $>3 \mathrm{~mm}$ ) were evaluated, K14-Angptl2 $\mathrm{Tg}$ developed papillomas 5 weeks earlier than did wild-type mice (top graph in Fig. 1C), and the average number of large papillomas was increased 3.7-fold in K14-Angptl2 Tg (bottom graph in Fig. 1C). Notably, there was no difference in the ratio of large papillomas to the total number between them (Fig. 1D). By 25 weeks after initiation of treatment, more than $80 \%$ of K14-Angptl2 Tg had developed malignant SCC, whereas only $50 \%$ of wild-type mice developed malignant SCC by this time point (top graph in Fig. 1E). The average number of SCC tumors was increased 5.44-fold in K14-Angptl2 Tg (bottom graph in Fig. 1E). As observed in Fig. 1F, the formation of SCC tumors was enhanced in K14-Angptl2 Tg. Histologic analysis confirmed that most large tumors were SCCs (Supplementary Fig. S7A and B). SCCs in K14-Angptl2 Tg were poorly differentiated and had lost their epithelial properties by acquisition of mesenchymal morphology. The malignant conversion rate, defined as the ratio of SCCs to the number of large papillomas, in K14Angptl2 Tg was greater than that calculated for wild-type mice (Fig. 1G). Thus, Angptl2 overexpression enhances susceptibility to "preneoplastic change" and "malignant conversion" in this model. Furthermore, we found that expression levels of the inflammatory marker $I L-1 \beta$ in both skin tissues after chemical treatment (Supplementary Fig. S8A) and papilloma (Supplementary Fig. S9A) of K14-Angptl2 Tg were significantly increased compared with wild-type mice, suggesting that Angptl2-associated inflammation may promote susceptibility to "preneoplastic change" and "malignant conversion" in this model.

\section{Angptl2 activation in tumor cells promotes metastasis to distant organs and lymph nodes}

Although lung metastasis was detected in both K14-Angptl2 $\mathrm{Tg}$ and wild-type mice, its frequency was significantly increased in K14-Angptl2 Tg (Fig. 2A). The severity of metastasis, as indicated by the number and area of metastatic lung tumor sites in the H\&E stained section, was greater in K14Angptl2 Tg compared with wild-type mice (Fig. 2B and C). Notably, $66.7 \%$ of K14-Angptl2 Tg showed metastasis to lung and more than $30 \%$ showed metastasis to both spleen and liver (Supplementary Fig. S10). In contrast, 17.6\% of wild-type mice showed metastasis only to lung but not to spleen or liver. K14Angptl2 Tg also showed increased frequency of metastasis to lymph nodes (Fig. 2D) and exhibited significantly enlarged lymph nodes compared with wild-type mice (Fig. 2E and F). Histologic analysis confirmed that enlarged lymph nodes were due to tumor cell metastasis rather than to inflammatory swelling (Fig. 2E). Finally, when we compared survival periods after development of malignant SCC, K14-Angptl2 Tg showed significantly shortened survival compared with wild-type mice (Fig. 2G). Expression levels of the inflammatory markers $I L-6$ and $I L-1 \beta$ in SCC of K14-Angptl2 Tg were also significantly increased compared with wild-type mice (Supplementary Fig. S11A), suggesting that Angptl2-associated inflammation might be attributable to increased tumor metastasis in K14-Angptl2 Tg.

\section{Suppressed carcinogenesis in skin tissues of Angptl2 knockout mice}

On the basis of our observation that Angptl2 overexpression enhances susceptibility to carcinogenesis and increases tumor metastasis, we asked whether Angptl2 deletion altered these pathologies. Therefore, we subjected Angptl2 KO and wild-type mice to the skin carcinogenesis regimen. We observed no papillomas or carcinoma in wild type or Angptl2 KO treated with DMBA alone or PMA alone (data not shown). When mice were treated with both chemicals, papilloma formation in Angptl2 KO was suppressed compared with wild-type mice (Fig. 3A). The average latency period for formation of skin papillomas in Angptl2 KO was 15 weeks after chemical application, compared with 10 weeks in wild-type mice (top graph in Fig. 3B). At 20 weeks after treatment, Angptl2 KO exhibited an average of 2.6 papillomas per mouse compared with 7.7 in wild-type mice (bottom graph in Fig. 3B). When only large papillomas were evaluated, Angptl2 KO developed large papillomas 4 weeks later than did wild-type mice (top graph in Fig. $3 \mathrm{C}$ ), and the average number of large papillomas was also decreased 0.25-fold in Angptl2 KO (bottom graph in Fig. 3C). Notably, there was no difference in the ratio of large papillomas to the total number between them (Fig. 3D). By 25 weeks after treatment, about $50 \%$ of wild-type mice had developed malignant SCC, whereas only $10 \%$ of Angptl2 KO developed malignant SCC by this time point (top graph in Fig. 3E). At 25 weeks after treatment, the average number of SCC lesions was decreased 0.14-fold in Angptl2 KO compared with wild-type mice (bottom graph in Fig. 3E). As shown in Fig. 3F, SCC development was significantly attenuated in Angptl2 KO. In contrast to SCCs in wild-type mice, SCCs in Angptl2 KO were histologically well-differentiated tumors and tended to be organized in epithelial structures (Supplementary Fig. S7C and D). The malignant conversion rate, defined as a ratio of SCC lesions to the number of large papillomas, was lower in Angptl2 KO than in wild-type mice (Fig. 3G). By contrast to findings in K14-Angptl2 Tg, expression levels of inflammatory markers in skin tissues before (Supplementary Fig. S5B) and after (Supplementary Fig. S8B) chemical treatment and in papillomas (Supplementary Fig. S9B) of Angptl2 KO were decreased compared with wild-type mice. Thus the absence of Angptl2induced inflammation may underlie attenuation of "preneoplastic change" and "malignant conversion" in carcinogenesis in Angptl2 KO. 


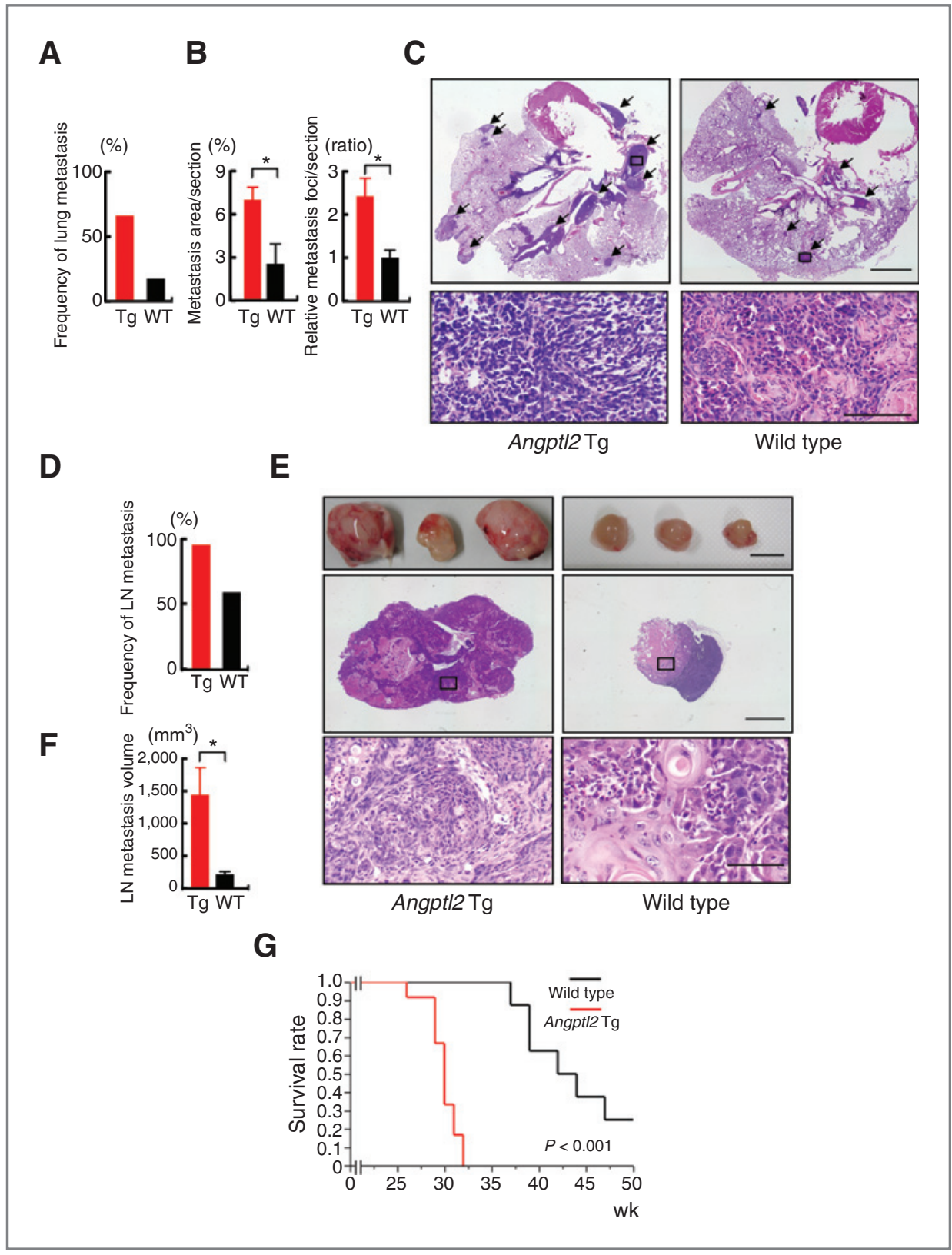

Figure 2. K14-Angpt/2 transgenic mice show enhanced metastasis and shortened survival. A, comparison of frequency of lung metastasis events between K14Angpt/2 $\mathrm{Tg}(n=21)$ and wild-type mice $(n=17)$. B, quantitative comparison of lung metastasis in K14-Angpt/2 Tg $(n=6)$ and wildtype mice $(n=5)$ on the basis of the area of tumor cells per area of lung section (left) and the relative number of metastatic foci per a lung section (right, wild-type data set at 1). C, H\&E-stained lung tissues of K14-Angpt/2 Tg and wild-type mice. Arrows indicate masses of metastatic tumor cells. Bottom panels are magnifications of squares in top panels. Scale bar, $2.5 \mathrm{~mm}$ (top), $100 \mu \mathrm{m}$ (bottom). D, comparison of frequency of metastasis to lymph nodes (LN) between K14-Angptl2 $\mathrm{Tg}(n=21)$ and wild-type mice $(n=17)$. E, photograph of gross appearance (top) and H\&E staining (middle and bottom) of lymph node from K14Angpt/2 Tg and wild-type mice. Bottom panels are magnifications of squares in middle panels. Scale bar, $10 \mathrm{~mm}$ (top), $4 \mathrm{~mm}$ (middle), $100 \mu \mathrm{m}$ (bottom). F, comparison of lymph node volume in K14-Angpt/2 $\operatorname{Tg}(n=6)$ and wild-type mice $(n=$ 6). G, Kaplan-Meier survival curves after initiation of chemical application of K14-Angpt/2 Tg $(n=12)$ and wild-type mice $(n=8$ $P<0.001$ by log-rank test). Data shown in $B$ and $F$ represent means \pm SEM. WT, wild type. ${ }^{*}, P<0.05$

\section{Angptl2 knockout mice show decreased lung and lymph node metastasis}

At 8 weeks after detection of the first SCC, $16.7 \%$ of wild-type mice showed metastasis to lung, whereas no metastases were seen in Angptl2 KO (Fig. 4A). Metastatic colonies of tumor cells were detected at multiple sites within the lung of wild-type mice, whereas none were seen in Angptl2 KO (Fig. 4B). Although metastasis to lymph nodes was detected in both genotypes, the frequency of metastasis was significantly lower in Angptl2 KO compared with wild-type mice (Fig. 4C). The severity of metastasis, as estimated by the size of metastatic lymph node sites, in Angptl2 KO was also significantly decreased relative to that seen in wild-type mice (Fig. 4D and E). Finally, survival of Angptl2 KO was significantly prolonged compared with that of wild-type mice (Fig. 4F). Expression of $I L-6$ and $I L-1 \beta$ in SCC of Angptl2 KO was also significantly decreased compared with that seen in wild-type mice (Supplementary Fig. S11B), suggesting that decreased tumor metastasis in Angptl2 KO may be due to the absence of Angptl2induced inflammation in SCC.

\section{Angptl2 derived from SCC contributes to tumor angiogenesis and lymphangiogenesis}

Tumor angiogenesis and lymphangiogenesis are essential for tumor growth and metastasis. Because we previously reported that Angptl2 activates endothelial cell motility (9), we asked whether the extent of SCC tumor vasculature induced by chemical carcinogenesis differed between K14-Angptl2 Tg 
A

At 18 weeks after the beginning of chemical application

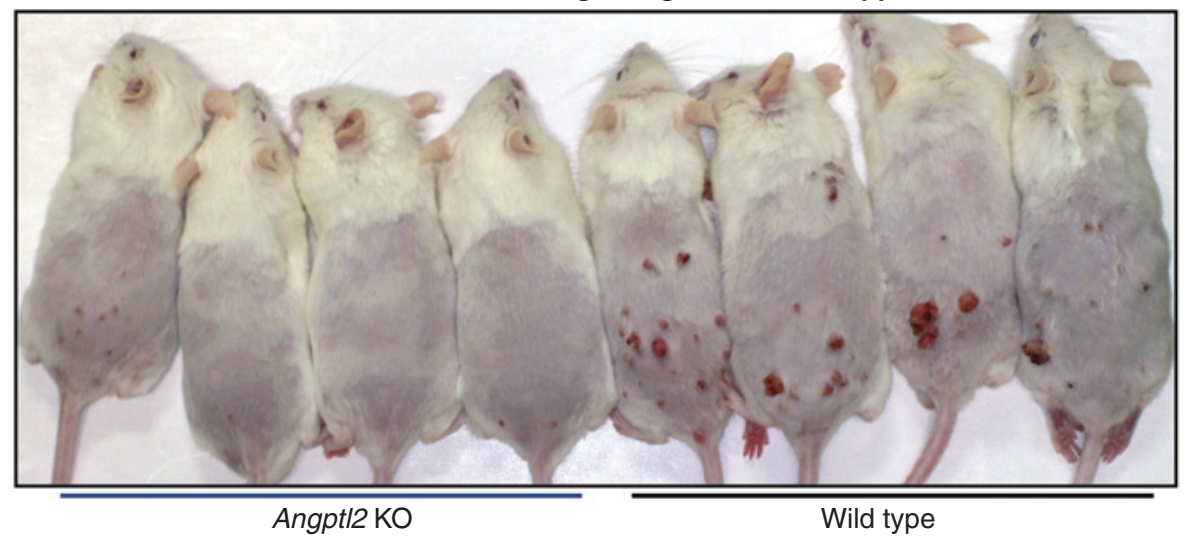

B

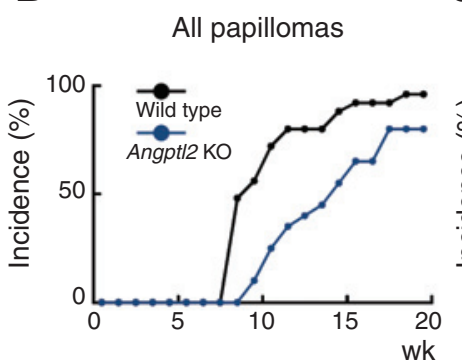

C

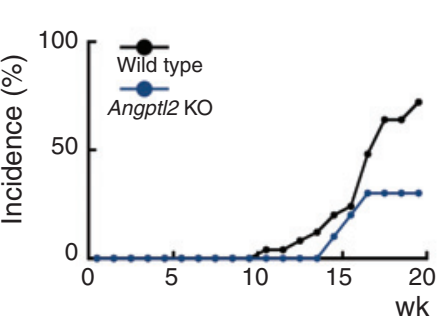

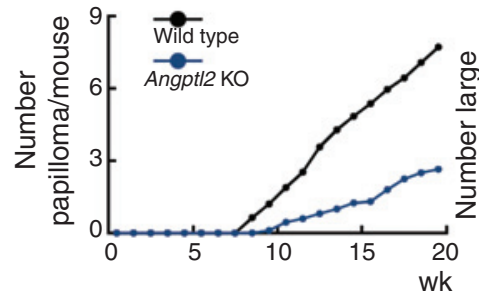

F

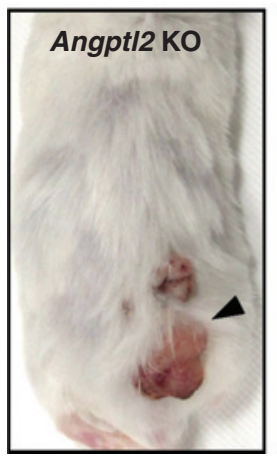

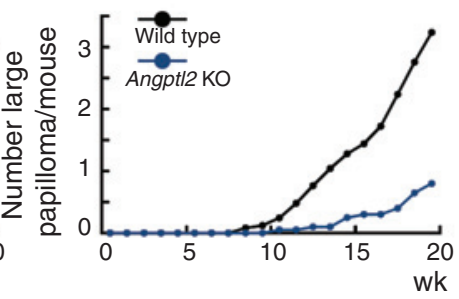

wk
D

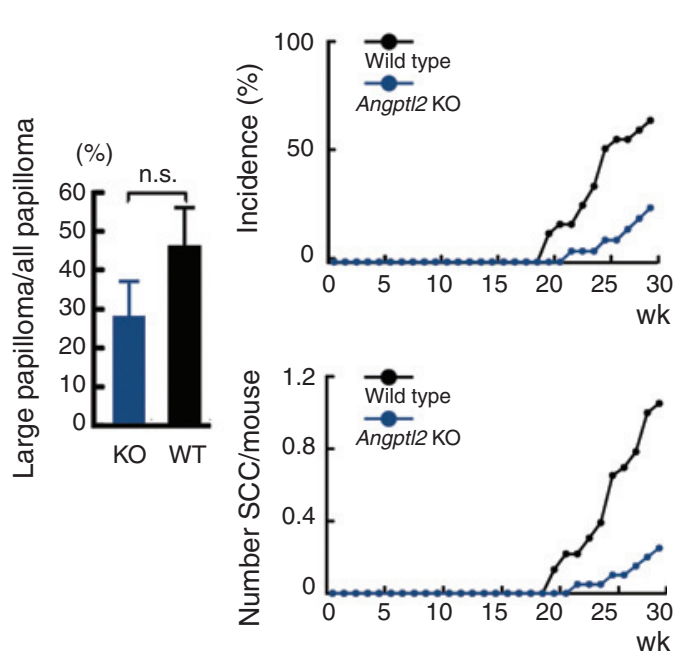

E

G

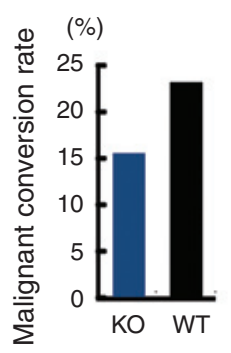

Figure 3. Angpt/2 knockout mice show suppressed and decreased skin carcinogenesis. A, photograph of skin of Angpt/2 KO (left) and wild-type mice (right) 18 weeks after initiation of chemical application. B and C, the incidence (top graphs) and number (bottom graphs) of all detectable (B) and large papillomas (C) on Angpt/2 KO ( $n=20$; blue circles) and wild-type mice $(n=25$; black circles) after initiation of chemical application. $P<0.01$ from weeks 10 to 20 (bottom graph in B), $P<0.05$ from weeks 14 to $16, P<0.01$ from weeks 17 to 20 (bottom graph in $\mathrm{C}$ ). $\mathrm{D}$, proportion of large compared with detectable papillomas in Angpt/2 KO versus wild-type mice. Data represent means \pm SEM. n.s., not significant. E, decreased incidence of SCC (top graph) and number of SCC per mouse (bottom graph, $P<0.05$ at weeks $24, P<0.01$ after weeks 25$)$ in Angpt/2 KO $(n=33$; blue circles) compared with wild-type mice $(n=34$; black circles). F, photograph of SCC on Angpt/2 KO (left 2 panels) and wild-type mice (right 2 panels) 8 weeks after first SCC diagnosis. Arrowheads indicate SCC. Scale bars, $10 \mathrm{~mm}$. G, malignant conversion rate of large papillomas to SCC in Angpt/2 KO and wild-type mice. WT, wild type. 


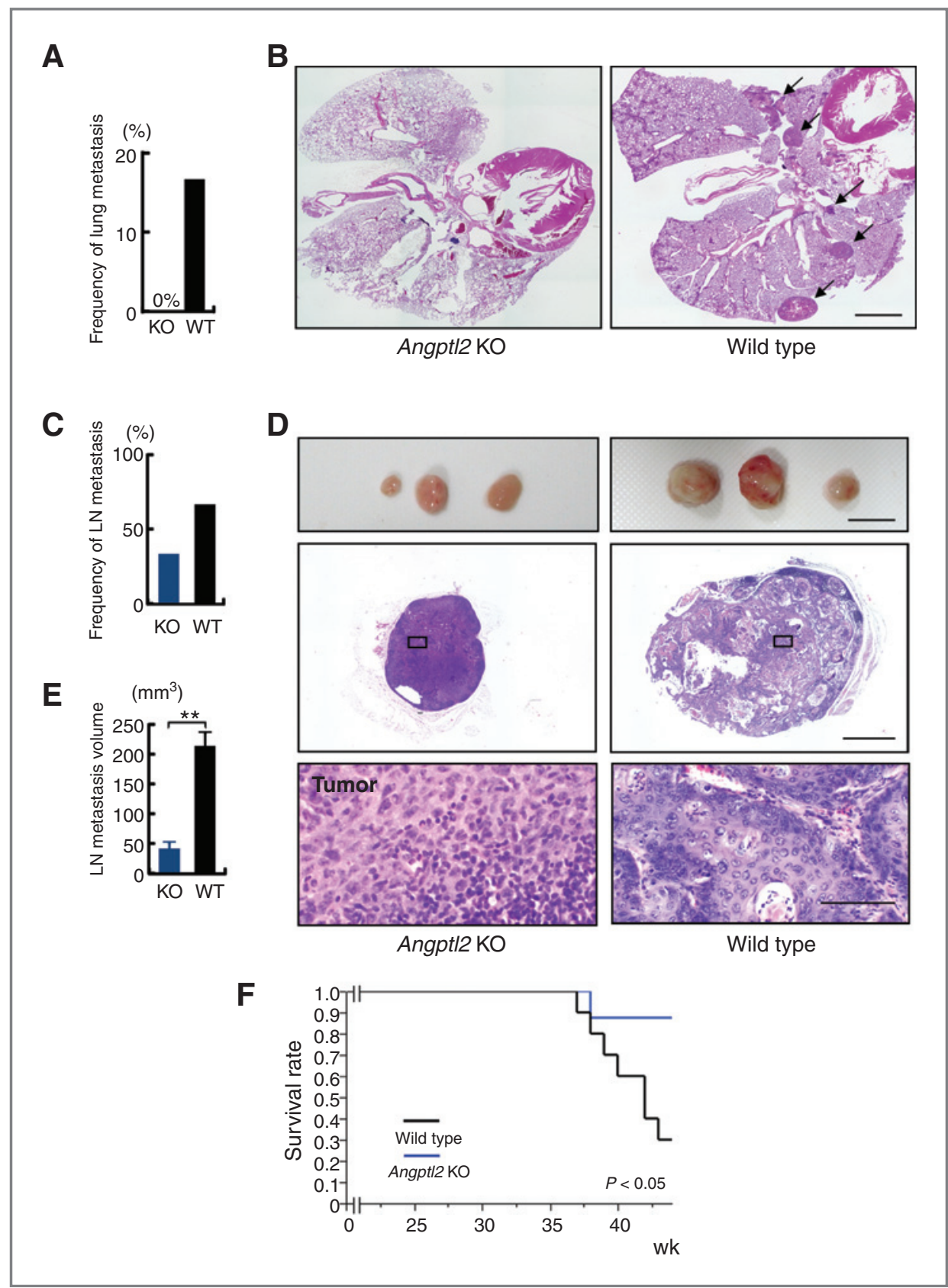

Figure 4. Angpt/2 knockout mice show decreased metastasis and prolonged survival. A, frequency of metastasis to distant secondary organs in Angpt/2 KO $(n=21)$ versus wild-type mice $(n=17)$. B $\mathrm{H} \& \mathrm{E}-$ stained lung tissues of Angpt/2 KO (left) and wild-type mice (right). Arrows indicate metastatic foci in wild-type. Scale bar, $2.5 \mathrm{~mm}$. C, frequency of metastasis to lymph nodes (LN) between Angpt/2 KO $(n=21)$ and wild-type mice $(n=$ 17). D, gross appearance (top) and H\&E staining (middle and bottom) of lymph nodes of Angpt/2 KO and wild-type mice. Bottom panels are magnifications of squares in middle panels. Scale bar, $10 \mathrm{~mm}$ (top), $2 \mathrm{~mm}$ (middle), $100 \mu \mathrm{m}$ (bottom). E, comparison of lymph node volume in K14-Angpt/2 Tg ( $=6)$ and wild-type mice $(n=6)$. Data represent means \pm SEM. ${ }^{* *}, P$ $<0.01$. F, Kaplan-Meier survival curves after initiating chemical application between Angpt/2 KO ( $=8)$ and wild-type mice ( $n=10 ; P<$ 0.05 by log-rank test). WT, wild type.

and wild-type mice. We found that blood vessels invading cancer tissues from surrounding tissues in K14-Angptl2 Tg were more prominent than those in wild-type mice (Fig. 5A). Quantitative analysis revealed that the area occupied by both CD31-positive blood vessels and LYVE-1-positive lymphatic vessels was significantly increased in cancer tissues of K14Angptl2 Tg compared with wild-type mice (Supplementary Fig. S12A and B), suggesting that Angptl2 enhances tumor vasculature. In contrast, the number of blood vessels invading tumor sites from surrounding tissues in Angptl2 KO seemed to be decreased compared with wild-type mice (Fig. 5B). The area occupied by both blood vessels and lymphatic vessels was quantitatively decreased in tumor tissues of Angptl2 KO versus wild-type mice (Supplementary Fig. S12C and D). The extent of Evans blue dye leakage from SCC tumors was markedly attenuated in Angptl2 KO compared with wild-type mice (Supplementary Fig. S13A), a finding confirmed quantitatively (Supplementary Fig. S13B).

\section{The epithelial-to-mesenchymal transition correlates} with Angpt12 expression levels

H\&E staining of SCC tumor cells in K14-Angptl2 Tg revealed neither an ordered structure nor tight cell-cell adhesion compared with wild-type mice (Supplementary Fig. S14A). 
Figure 5. Angptl2 derived from cancer cells contributes to tumor angiogenesis and

lymphangiogenesis. A, photograph of vasculature in primary tumors induced by chemical skin carcinogenesis of K14-Angpt/2 Tg and wild-type mice. Right 2 panels are magnifications of squares in left panels, respectively. Scale bar, $10 \mathrm{~mm}$ (left), $10 \mathrm{~mm}$ (right). B, photograph of vasculature of primary tumors induced by chemical skin carcinogenesis of Angpt/2 KO and wild-type mice. Right 2 panels are magnifications of squares in left panels, respectively. Scale bar, 10 $\mathrm{mm}$ (left), $10 \mathrm{~mm}$ (right).

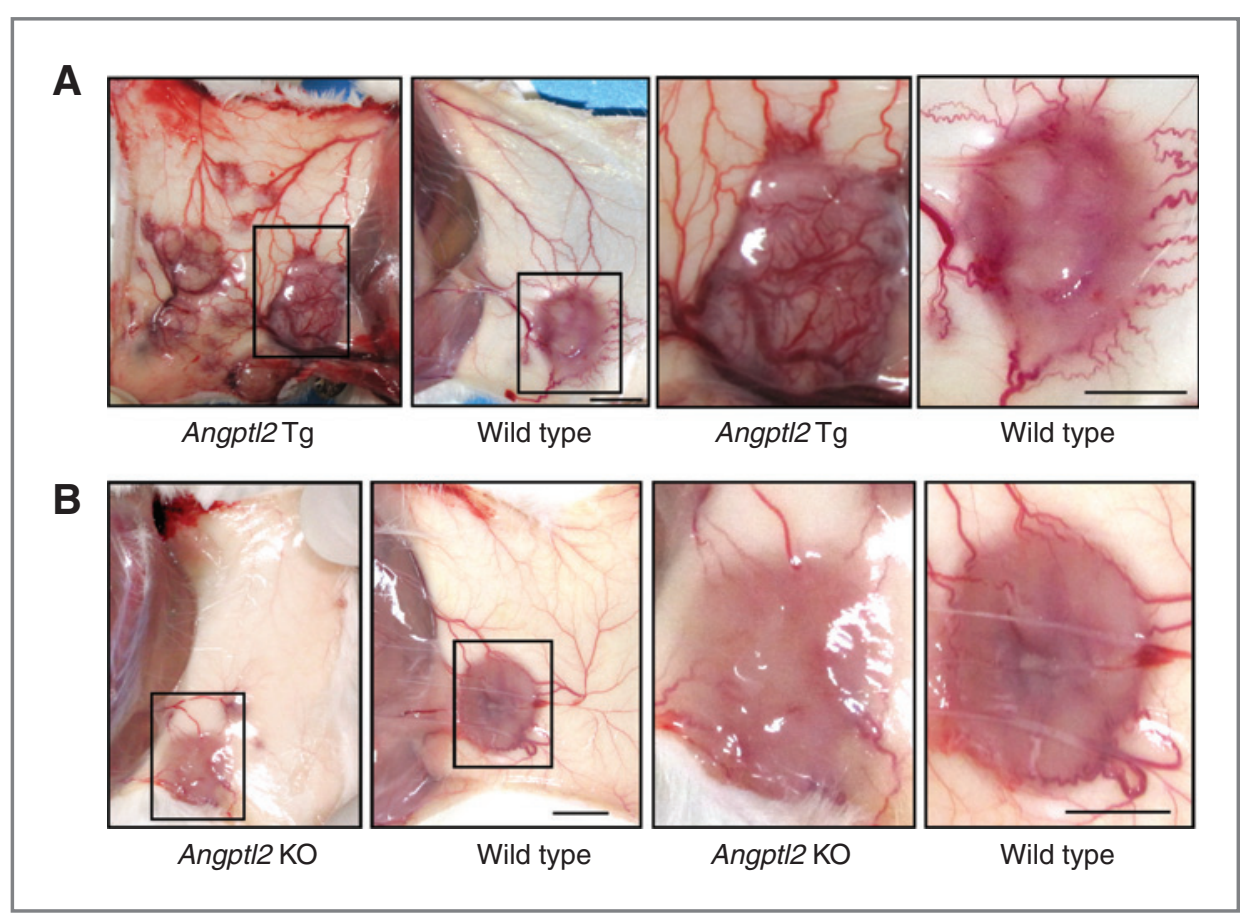

Instead, tumor cells showed an irregular structure, appeared more elongated, and were not uniform in composition or density. Because a critical event in tumor metastasis is a decrease in cancer cell adhesion through acquisition of mesenchymal phenotypes and invasive properties, we asked whether Angptl2 increases the epithelial-to-mesenchymal transition (EMT) in SCC. Relative to wild-type mice, increased $N$-cadherin, Slug, and Snail mRNA, as mesenchymal markers and decreased E-cadherin mRNA, as an epithelial indicator were observed in SCC of K14-Angptl2 Tg (Supplementary Fig. S15A). Immunohistochemical analysis also revealed increased $\mathrm{N}$-cadherin expression, decreased E-cadherin expression, and increased nuclear levels of Slug and Snail in SCC of K14-Angptl2 Tg compared with wild-type mice (Fig. 6A), suggesting that constitutive activation of Angptl2 signaling may underlie EMT. Conversely, H\&E staining showed that SCC tumor cells in Angptl2 KO formed a sheet (Supplementary Fig. S14B) and abutted each other in a uniform array. Furthermore, decreased $N$-cadherin, Slug, and Snail mRNA and increased E-cadherin mRNA were observed in SCC of Angptl2 KO compared with wild-type mice (Supplementary Fig. S15B). Immunohistochemical analysis also revealed decreased E-cadherin expression and increased nuclear Slug and Snail in SCC from wildtype mice, whereas SCC of Angptl2 KO exhibited primarily epithelial phenotypes and showed little nuclear translocation of Slug or Snail (Fig. 6B). TGF- $\beta /$ Smad signaling plays a pivotal role in promoting EMT (21-23). To investigate mechanisms underlying activation of EMT seen in the presence of Angptl2 overexpression, we analyzed activation of this pathway. We observed increased expression of TGF- $\beta 1, T G F-\beta 2, T G F-\beta R 1$, and TGF- $\beta R 2$ mRNAs in SCC of K14-Angptl2 Tg compared with wild-type mice (Supplementary Fig. S15C). Conversely, we observed decreased relative expression of those genes in SCC of Angptl2 KO mice (Supplementary Fig. S15D). In addition, phosphorylation of Smad2, a TGF- $\beta$ effector, was significantly decreased in SCC of Angptl2 KO, whereas Smad2 phosphorylation was significantly increased in SCC of K14-Angptl2 Tg compared with controls (Fig. 6C). These findings suggested that Angptl2 promotes EMT via activating the TGF- $\beta$-Smad pathway.

\section{Discussion}

Here, we found that skin tissues expressing abundant Angptl2 exhibit high carcinogenic potential in a chemically induced skin SCC mouse model through increased susceptibility to both "preneoplastic change" and "malignant conversion", although abundant Angptl2 expression in skin tissues could not cause SCC. Furthermore, Angptl2 derived from SCC increased cancer cell metastasis to both distant secondary organs and lymph nodes, presumably, by promoting both EMT in cancer cells and tumor angiogenesis and lymphangiogenesis.

The initiating oncogenic mutation within a normal cell is essential for "preneoplastic change." It is also important that cells harboring that mutation acquire the proliferation and survival capacity to allow accumulation of additional mutations (24-26). When an oncogenic mutation occurs within a normal cell, DNA repair mechanisms often avert carcinogenesis. However, increased accumulation of reactive oxygen species (ROS) and reactive nitrogen intermediates because of chronic inflammation can inactivate DNA repair enzymes (27). In this regard, both chronic inflammatory status and ROS levels in mouse skin tissues are positively correlated with Angptl2 


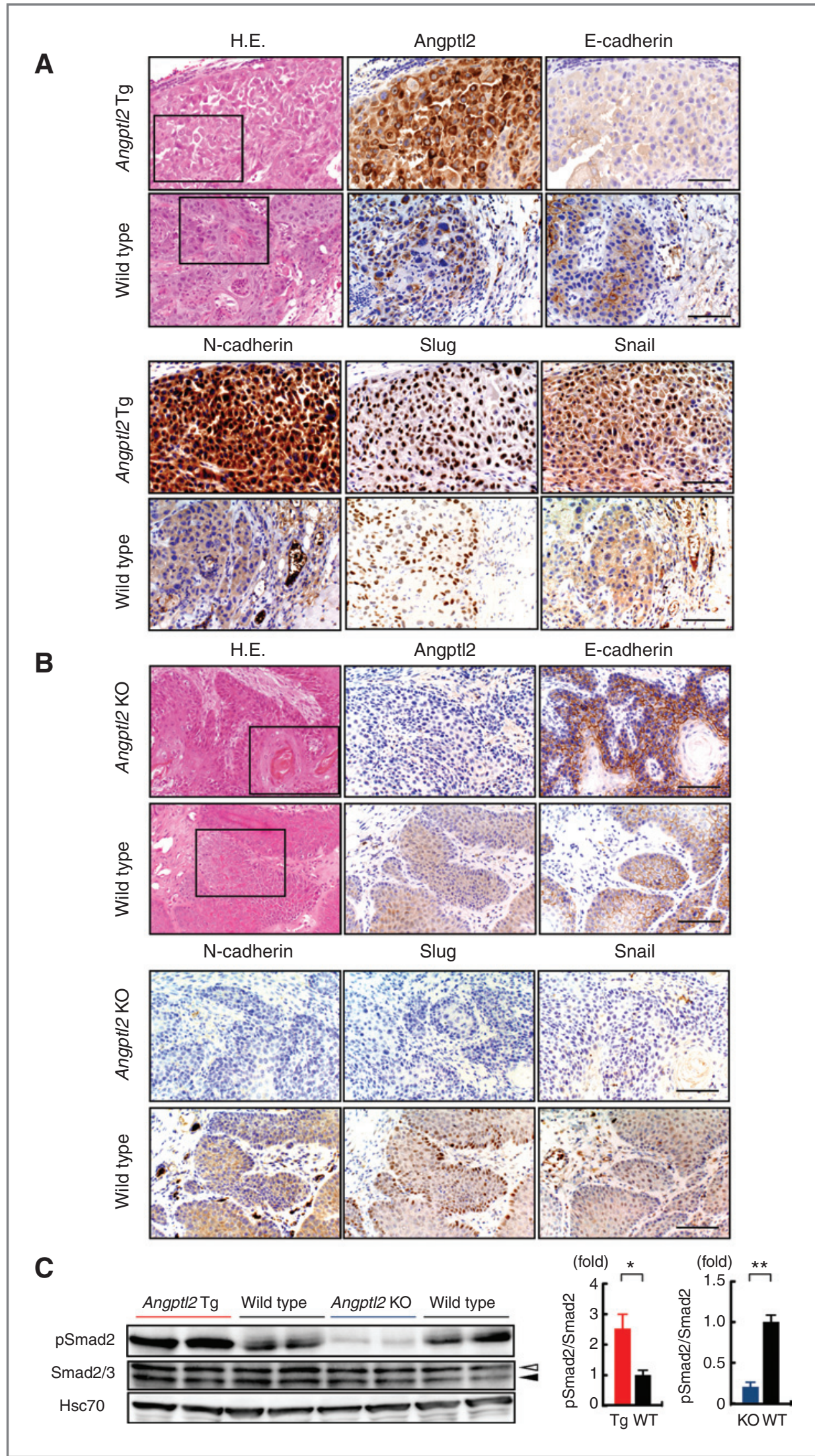

Figure 6. Angptl2 expression levels are correlated with the epithelialto-mesenchymal transition. A and $B, H \& E$ staining and immunohistochemical staining for Angptl2, E-cadherin, N-cadherin, Slug, and Snail in primary tumor tissue of K14-Angpt/2 Tg and wildtype mice (A) and Angpt/2 KO and wild-type mice (B). C, left, Western blotting analysis of phosphorylated Smad2, total Smad2/3, and Hsc70 in primary tumor tissue. Open and closed arrowheads indicate Smad2 and Smad3, respectively. Right, quantitative analysis of Western blotting analysis $(n=3)$. Scale bars, $100 \mu \mathrm{m}$. Data are expressed as means \pm SEM *, $P<0.05 ;{ }^{* *}, P<0.01$ compared with wild-type mice. 
expression levels (Supplementary Fig. S16), suggesting that Angptl2-associated inflammation creates a microenvironment conducive to DNA damage and genomic instability. Normal skin stem cells or transit amplifying cells are more susceptible to mutation than are differentiated cells (2). An inflammatory microenvironment reportedly increases the number of stem cells and transit amplifying cells (27), suggesting that Angptl2associated inflammatory changes in skin tissues might increase these stem cell populations. However, we observed no difference in the number of normal skin stem cells as estimated by a long-time pulse of bromodeoxyuridine incorporation experiment (28) among Angptl2 KO, K14-Angptl2 Tg, and their respective wild-type littermates (data not shown). Taken together, we propose that Angptl2 increased susceptibility to both "preneoplastic change" and "malignant conversion" is more likely due to differences in the microenvironment conducive to maintenance of oncogenic DNA mutations and accumulation of additional mutations.

Although we found no papillomas or carcinoma at any time point in either K14-Angptl2 Tg, Angptl2 KO, or wild-type mice treated with PMA alone (data not shown), treatment with PMA only did induce Angptl2 expression in wild-type mice (Supplementary Fig. S6). In analysis of human subjects, Angptl2 expression in skin tissues exposed to sunlight was increased compared with tissues not exposed to sun (Supplementary Fig. S17), suggesting Angptl2 induction in skin cells by sunburn might increase cancer susceptibility. This finding is consistent with the well-established idea that sunburn increases the risk of skin carcinogenesis because of DNA damage and inflammation $(19,29)$. Most solid malignancies appear in older subjects, and aging or cell senescence is postulated to function as cancer promoters that act through inflammatory mechanisms. Accordingly, Angptl2 expression in regions not exposed to sun was elevated in parallel with aging in human subjects (Supplementary Fig. S18). Sunburn- or aging-induced Angptl2 and its related chronic inflammation in skin tissue might increase susceptibility to skin carcinogenesis.

Angptl2 is reportedly a putative tumor suppressor in ovarian cancer (15). In that report, lack of Angptl2 immunoreactivity in tumor cells was associated with poorer overall survival in stage I and II disease, whereas Angptl2 positivity was significantly

\section{References}

1. Chiang AC, Massague J. Molecular basis of metastasis. N Engl J Med 2008;359:2814-23.

2. Grivennikov SI, Greten FR, Karin M. Immunity, inflammation, and cancer. Cell 2010;140:883-99.

3. de Martel C, Franceschi S. Infections and cancer: established associations and new hypotheses. Crit Rev Oncol Hematol 2009;70:183-94.

4. Takahashi $\mathrm{H}$, Takahashi I, Honma M, Ishida-Yamamoto A, lizuka H. Prevalence of metabolic syndrome in Japanese psoriasis patients. $J$ Dermatol Sci 2010;57:143-4.

5. Dostert C, Petrilli V, Van Bruggen R, Steele C, Mossman BT, Tschopp $\mathrm{J}$. Innate immune activation through Nalp3 inflammasome sensing of asbestos and silica. Science 2008;320:674-7.

6. Kant P, Hull MA. Excess body weight and obesity-the link with gastrointestinal and hepatobiliary cancer. Nat Rev Gastroenterol Hepatol 2011;8:224-38.

7. Calle EE. Obesity and cancer. BMJ 2007;335:1107-8. associated with a poorer survival in stage III and IV disease. The latter findings are consistent with our own, suggesting that Angptl2 is critical for tumor progression and metastasis. Here, we did not observe a tumor suppressor function for Angptl2 in skin cells. Whether Angptl2 function differs in various tumor cell types remains to be determined.

This study shows that Angptl2 expression increases carcinogenesis through enhanced susceptibility to both "preneoplastic change" and "malignant conversion." Chronic inflammatory status during carcinogenesis was markedly different among K14-Angptl2 Tg, Angptl2 KO, and wild-type mice, and it depended on Angptl2 expression levels. This suggested that Angptl2-associated chronic inflammation is important for cancer susceptibility. Angptl2 derived from tumor cells significantly increased distant secondary organ metastasis and lymph node metastasis through acquisition of EMT-related invasive properties, in addition to promoting tumor angiogenesis and lymphangiogenesis (Supplementary Fig. S19). Overall, our findings suggest that Angptl2 represents a new target for prevention of these activities in preneoplastic lesions and cancers that express Angptl2.

\section{Disclosure of Potential Conflicts of Interest}

No potential conflicts of interest were disclosed.

\section{Acknowledgments}

The authors thank Mss. S. Iwaki, O. Takahashi, and M. Nakata for technical assistance.

\section{Grant Support}

This work was supported by grants-in-aid for Scientific Research on Priority Areas from the Ministry of Education, Culture, Sports, Science and Technology of Japan, by the Japan Society for the Promotion of Science (ISPS) through its Funding Program for Next Generation World-Leading Researchers (NEXT Program), and by grants from the Takeda Science Foundation, the Mitsubishi Foundation, and the Tokyo Biochemical Research Foundation.

The costs of publication of this article were defrayed in part by the payment of page charges. This article must therefore be hereby marked advertisement in accordance with 18 U.S.C. Section 1734 solely to indicate this fact.

Received May 26, 2011; revised October 10, 2011; accepted October 24, 2011; published OnlineFirst October 31, 2011. 
synovial chronic inflammation in rheumatoid arthritis. Am J Pathol 2010;176:2309-19.

13. Avraamides CJ, Garmy-Susini B, Varner JA. Integrins in angiogenesis and lymphangiogenesis. Nat Rev Cancer 2008;8:604-17.

14. Desgrosellier JS, Cheresh DA. Integrins in cancer: biological implications and therapeutic opportunities. Nat Rev Cancer 2010;10:9-22.

15. Kikuchi R, Tsuda H, Kozaki K, Kanai Y, Kasamatsu T, Sengoku K, et al. Frequent inactivation of a putative tumor suppressor, angiopoietin-like protein 2, in ovarian cancer. Cancer Res 2008;68:5067-75.

16. Yuspa SH, Døugosz AA, Cheng CK, Denning MF, Tennenbaum T, Glick $A B$, et al. Role of oncogenes and tumor suppressor genes in multistage carcinogenesis. J Invest Dermatol. 1994;103:90S-5S.

17. Hawighorst $T$, Velasco $P$, Streit $M$, Hong $Y K$, Kyriakides TR, Brown LF, et al. Thrombospondin-2 plays a protective role in multistep carcinogenesis: a novel host anti-tumor defense mechanism. EMBO $J$ 2001;20:2631-40.

18. Hawighorst $T$, Oura $H$, Streit $M$, Janes $L$, Nguyen L, Brown LF, et al. Thrombospondin-1 selectively inhibits early-stage carcinogenesis and angiogenesis but not tumor lymphangiogenesis and lymphatic metastasis in transgenic mice. Oncogene 2002;21:7945-56.

19. Yuspa SH. The pathogenesis of squamous cell cancer: lessons learned from studies of skin carcinogenesis. J Dermatol Sci 1998;17:1-7.
20. Yuspa SH. Overview of carcinogenesis: past, present and future. Carcinogenesis 2000;21:341-4.

21. Ikushima $\mathrm{H}$, Miyazono K. TGF- $\beta$ signalling: a complex web in cancer progression. Nat Rev Cancer 2010;10:415-24.

22. Massague J. TGF- $\beta$ in Cancer. Cell 2008;134:215-30.

23. Bierie B, Moses HL. Tumour microenvironment: TGF- $\beta$ : the molecular Jekyll and Hyde of cancer. Nat Rev Cancer 2006;6:506-20.

24. Knudson AG. Two genetic hits (more or less) to cancer. Nat Rev Cancer 2001;1:157-62.

25. Hanahan D, Weinberg RA. Hallmarks of cancer: the next generation. Cell 2011;144:646-74.

26. Fearon $E R$, Vogelstein $B$. A genetic model for colorectal tumorigenesis. Cell 1990;61:759-67.

27. Colotta F, Allavena P, Sica A, Garlanda C, Mantovani A. Cancer-related inflammation, the seventh hallmark of cancer: links to genetic instability. Carcinogenesis 2009;30:1073-81.

28. Cotsarelis G, Sun TT, Lavker RM. Label-retaining cells reside in the bulge area of pilosebaceous unit: implications for follicular stem cells, hair cycle, and skin carcinogenesis. Cell 1990;61: 1329-37.

29. Alam M, Ratner D. Cutaneous squamous-cell carcinoma. N Engl J Med 2001;344:975-83. 


\section{Cancer Research}

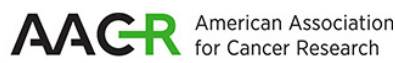

The Journal of Cancer Research (1916-1930) | The American Journal of Cancer (1931-1940)

\section{Angiopoietin-like Protein 2 Is an Important Facilitator of Inflammatory Carcinogenesis and Metastasis}

Jun Aoi, Motoyoshi Endo, Tsuyoshi Kadomatsu, et al.

Cancer Res 2011;71:7502-7512. Published OnlineFirst October 31, 2011.

Updated version Access the most recent version of this article at: doi:10.1158/0008-5472.CAN-11-1758

Supplementary Access the most recent supplemental material at:

Material http://cancerres.aacrjournals.org/content/suppl/2011/10/31/0008-5472.CAN-11-1758.DC1

Cited articles This article cites 28 articles, 7 of which you can access for free at:

http://cancerres.aacrjournals.org/content/71/24/7502.full.html\#ref-list-1

Citing articles This article has been cited by 21 HighWire-hosted articles. Access the articles at: /content/71/24/7502.full.html\#related-urls

E-mail alerts Sign up to receive free email-alerts related to this article or journal.

Reprints and To order reprints of this article or to subscribe to the journal, contact the AACR Publications Department a Subscriptions pubs@aacr.org.

Permissions To request permission to re-use all or part of this article, contact the AACR Publications Department at permissions@aacr.org. 